\section{Injury severity in relation to seatbelt use}

To the Editor: I read the article by Van Hoving et al. ${ }^{[1]}$ with great interest. Two interesting aspects of the study are that it included patients in the prehospital setting, and involved both the Division of Emergency Medicine and the Department of Civil Engineering at their institution (Stellenbosch University, Western Cape, South Africa). The authors acknowledge that it is a pilot study with many limitations. Nevertheless, important lessons can be learned from it that may improve the design and performance of future studies.

First, it is known that using seatbelts reduces injury severity and death. ${ }^{[2,3]}$ However, owing to the complexity of road traffic injuries, it is important to include the maximum number of injured patients, depending on the inclusion/exclusion criteria, to strengthen the analysis and permit a proper multivariate analysis to answer the research question properly. It is not clear to me, for example, why patients involved in taxi accidents $(n=94)$ were excluded from the present study. ${ }^{[1]}$ This is an unusual reason for exclusion, especially when only 107 patients were included in the prehospital phase.

Second, study of the biomechanics of road traffic collisions (RTCs) in more detail, including ejection from the vehicle, is essential. The authors cited our study reporting a significant effect of seatbelt use in reducing injury severity when we performed a univariate comparison between patients who had used seatbelts and those who had not. ${ }^{[4]}$ However, once we included other important factors using a general linear model, ${ }^{[5]}$ we found that the mechanism of the collision and vehicle speed were the most important factors affecting the severity of RTC injuries, while the effect of seatbelts became non-significant, indicating that the main effect of seatbelt use was to reduce ejection from the vehicle. ${ }^{[5]}$ Injury severity among patients who were ejected was double that in cases in which the vehicle sustained lateral impact or rolled over. ${ }^{[5]}$ I note that ejection was not included in Van Hoving et al.'s ${ }^{[1]}$ study.

Third, there were many missing hospital data in the study ${ }^{[1]}$ We have addressed this issue by appointing a full-time researcher to collect data prospectively from hospitals on a daily basis. This has increased the completeness of data on seatbelt use to $98 \%$ in our Road Traffic Collision Registry. ${ }^{[4]}$

Finally, I congratulate the authors on their important study and hope that they will find these comments useful.

\section{Fikri M Abu-Zidan}

Professor, Consultant Trauma and Acute Care Surgeon and Medical Statistician, Department of Surgery, College of Medicine and Health Sciences, United Arab Emirates University, Al-Ain, United Arab Emirates

fabuzidan@uaeu.ac.ae

1. Van Hoving DJ, Hendrikse C, Gerber RJ, Sinclair M, Wallis LA. Injury severity in relation to seatbel use in Cape Town, South Africa: A pilot study. S Afr Med J 2014;(7)104:488-492. [http://dx.doi. org/10.7196/SAMJ.7933

2. Abbas AK, Hefny AF, Abu-Zidan FM. Seatbelts and road traffic collision injuries. World J Emerg Surg 2011:6:18. [http://dx.doi.org/10.1186/1749-7922-6-18]

3. Abbas AK, Hefny AF, Abu-Zidan FM. Seatbelt compliance and mortality in the Gulf Cooperation Council countries in comparison with other high-income countries. Ann Saudi Med 2011;31:347-350. [http://dx.doi.org/10.4103/0256-4947.83208]

4. Abu-Zidan FM, Abbas AK, Hefny AF, Eid HO, Grivna M. Effects of seat belt usage on injury pattern and outcome of vehicle occupants after road traffic collisions: Prospective study. World J Surg 2012;36(2):255-259. [http://dx.doi.org/10.1007/s00268-011-1386-y]

5. Abu-Zidan FM, Eid HO. Factors affecting injury severity of vehicle occupants following road traffic collisions. Injury (in press). [http://dx.doi.org/10.1016/j.injury.2014.10.066]

\section{Driving innovation, leadership and change at Groote Schuur Hospital, Cape Town, South Africa}

To the Editor: Globally, cost-effective and equitable delivery of healthcare is becoming a challenge. In the South African (SA) context, balancing the burden of disease and the patient load within available resources, while still maintaining quality of care, is becoming unsustainable. Our public healthcare services need to support a growing population of uninsured citizens together with immigrants from elsewhere in Africa seeking care, and while the reasons for the problem are both economic and related to health service design and delivery, it is compounded by an ageing population, a rising burden of infectious and chronic diseases and the global shortage of adequately skilled healthcare workers. The leaders of today will need to do something different in order to avoid collapse of the system.

Such a change in strategy comes with the realisation that there is no quick fix, and the transformation process will take time to embed and institutionalise. It is not a single event, but part of an overarching strategy. At the core of such a transformational strategy is the need to maximise the value for the patient. However, this focus should not deflect from the need to ensure that the staff who provide the service have the necessary resources and a safe and pleasant environment in which to perform their duties.

Groote Schuur Hospital (GSH) is a central hospital in the Western Cape, SA, and has embraced the Western Cape Department of Health's vision of 'Access to patient-centred, quality care', which is enacted through the values of caring, competence, accountability, integrity, responsiveness and respect. With this foundation, facing the ongoing challenges means that we cannot just continue with business as usual. In taking the hospital forward into the 21st century, the GSH management team adopted a theme of leadership, innovation and change as part of a journey to lead the institution towards excellence in healthcare. Change is required to meet the demands of today's environment, and this change can be effected through appropriate leadership and driving innovation with a view to improving the quality of care provided. For these transformational processes, we need people to address these changes and paint a picture of the world of tomorrow.

In 1967, the innovative accomplishment of the first human-tohuman heart transplant gave GSH international status. There have been many similar achievements since then, some of which have gone unnoticed. While they may not attract international acclaim, the innovations - whether related to process, service, quality or strategy have all contributed to improving the quality of patient care provided. Transformation through new ideas must come from within, and every stakeholder in the healthcare system has a role to play.

With this in mind, GSH and the Faculty of Health Sciences at the University of Cape Town (UCT) have partnered with UCT's Bertha Centre for Social Innovation and Entrepreneurship to catalyse staffled healthcare innovation. The GSH Facility Board has generously donated the funding for this Groote Schuur Hospital Innovations Programme (GSHIP). This is the first time an African healthcare institution has pursued such an initiative, and we are proud to be leading the way.

For the purposes of the programme, we have defined 'innovation' as simply a new and different solution to existing challenges. We have capitalised on the fact that our 3762 staff members have the intellectual and creative capital to think differently and develop bold solutions that can transform the way in which we deliver healthcare at GSH. 
The GSHIP was officially launched on 6 October 2014. Building up to this, all staff were engaged to help them understand the challenges faced. Based on feedback received, the following eight major challenges were identified for innovation:

\section{Using waiting time more effectively}

How could we design a better experience for patients waiting for treatment across the hospital? 'Waiting' does not have to be a waste of time, but can become an opportunity to address patients differently. Innovative solutions could include:

- Improving the waiting experience

- Reducing waiting times

- Addressing the health and wellbeing of patients while they are waiting.

\section{Sustaining a culture of care and dignity}

How could we support staff to deliver compassionate care all the time? Innovative solutions could include:

- Developing a caring culture model for teams

- Staff support to deal with frustrations

- Catalyse a wider culture change.

\section{Tracking and communicating}

What if we had better visibility of the patient's experience, e.g. waiting times, ward stock levels, free beds, theatre availability, and how well our patients are doing in real time? Innovative solutions could include:

- Improve data and information collection

- Sharing of data

- Effective use of data to reduce bottlenecks in care.

\section{Patient records and notes}

What if we had a better system of keeping track of our patient's records, inputting data and ultimately spending less time with administrative duties and more time with our patients? Innovative solutions could include:

- Reduce time spent searching and waiting for folders

- Make record management more user friendly

- Make records more applicable across departments and teams.

\section{More efficient entry and exit}

How could we improve the referral process, appointment bookings and quicker, safe discharge? Innovative solutions could include:
- Improve the system for patient appointments

- Improve the referral process

- Improve the discharge process

- Ensure that patients have the support they need when discharged home.

\section{Improve care for specific patient groups}

How could we radically improve the experience of our adolescent or tuberculosis patients waiting for care, and the quality and safety of the care they receive? Innovative solutions could include:

- Be centred around the needs of each group

- Improve their experience of care

- Improve clinical outcomes.

\section{Working better with district health services (DHSs)}

How could we support the DHSs, so that our patients can be treated near their homes and avoid unnecessary trips to the hospital? Innovative solutions could include:

- Smoother transition from hospital to community services

- Improved communication processes.

\section{Boost volunteer resources}

How could we help families and volunteers to play a more central role in caring for our patients? Innovative solutions could include:

- Building the skills of families and volunteers

- Develop opportunities for progress.

All staff have been given the opportunity to submit proposals with their innovative ideas. These will be presented to a panel of judges in January, and the selected winners will be awarded funds to implement their project with the aim of completion in October 2015. A space has been provided in GSH that will be transformed into an Innovation Hub, and the partners from the Bertha Centre will provide the expertise to assist innovators to structure their ideas into formal proposals.

With the success of this project, it is hoped that GSH can encourage the scalability of the innovative ideas to other public health facilities in the country.

\section{Bhavna Patel}

CEO, Groote Schuur Hospital, Cape Town, South Africa bhavna.patel@westerncape.gov.za

S Afr Med J 2015;105(1):5-6. DOI:10.7196/SAMJ.9209 